\title{
STELLAR WINDS IN HIGH-MASS X-RAY BINARIES
}

\author{
LEX KAPER \\ European Southern Observatory, Karl Schwarzschild Straße 2, \\ D-85748 Garching bei München, B.R. Deutschland \\ I: lkaper@eso.org
}

\begin{abstract}
High-mass X-ray binaries (HMXBs) represent an important stage in the evolution of massive binary systems. The compact object (in most cases an X-ray pulsar) not only provides information on the orbital and stellar parameters, but also probes the stellar wind of the massive companion, an OB supergiant or Be star. The X-ray luminosity directly depends on the density and the velocity of the wind at the orbit of the X-ray source. Important constraints on the stellar-wind structure can be set by studying the orbital modulation of UV P-Cygni profiles. In this paper different aspects of the interactive wind-accretion process are highlighted, such as the highly variable X-ray luminosity, the influence of the $\mathrm{X}$-rays on the radiative acceleration of the wind inside the ionization zone, and the large-scale structures that trail the X-ray source in its orbit.
\end{abstract}

Key words: stars: massive binaries - winds - X-ray sources

\section{Massive binary evolution}

Massive stars have strong stellar winds, driven by radiation pressure in numerous spectral lines. Since the pioneering work of Lucy \& Solomon (1970) and Castor, Abbott \& Klein (1975), radiation-driven wind theory is well developed and capable of explaining the mass-loss rates $\left(10^{5}-10^{6} \mathrm{M}_{\odot} / \mathrm{yr}\right)$ and terminal velocities $(1000-3000 \mathrm{~km} / \mathrm{s})$ in quantitative agreement with the observations (e.g., Kudritzki \& Hummer 1990). Stellar winds have important consequences for massive star evolution: phases of very high mass-loss rates (e.g., the luminous blue variable (LBV) stage) are believed to cause the total expulsion of the star's hydrogen-rich mantle, leading to the formation of a Wolf-Rayet star (see e.g., Maeder, this volume).

In massive close binary systems, mass loss due to mass transfer can play an even more significant role regarding the evolution of the binary components (for an excellent review on this subject, see van den Heuvel 1994). During phases of Roche-lobe overflow the primary star loses many solar masses and becomes a helium star, while the secondary star receives the hydrogenrich material and becomes the most massive star. The mass-gaining star adapts itself to its new mass on a thermal timescale and obtains an internal structure very similar to that of a single star of identical mass (Hellings 1983, Pols 1993). As the helium star is normally identified with a Wolf-Rayet star, in this stage the system has become a WR+O binary.

An important consequence of the mass transfer is that, after the supernova explosion of the primary star, the system remains bound because less than half of the total mass left the system (Blaauw 1961). From that moment 
on the compact remnant, a neutron star or black hole, orbits the secondary massive star. When the secondary evolves into a supergiant, it develops a strong radiation-driven wind; accretion of wind material onto the compact object results in the production of X-rays. Now we observe a high-mass Xray binary (HMXB). The $\mathrm{X}$-ray luminosity resulting from wind accretion is a function of the density and relative velocity of the wind at the orbit of the compact star (Bondi \& Hoyle 1944):

$$
L_{X} \sim \rho v_{\text {rel }}^{-3}
$$

For normal wind densities and velocities an X-ray luminosity of $10^{36-37} \mathrm{erg} / \mathrm{s}$ is easily obtained.

HMXBs are divided into two classes: one where the secondary star has become a Be star (containing 30 systems), the other with an OB supergiant as the secondary star ( $\sim 15$ known $)$. In Be/X-ray binaries often a transient $\mathrm{X}$-ray source is found, explained by the fact that a high (detectable) X-ray luminosity is only expected when the compact star crosses the dense equatorial disk of the Be star (van den Heuvel \& Rappaport 1987). By measuring the time interval between two successive "bursts" in X-ray luminosity, the orbital period of the system can be derived. The characteristic high rotation speed of the Be star is, in this scenario, a natural consequence of the increase of the star's total angular momentum due to the mass transfer which had occurred. In the following we will, however, concentrate on the OB supergiant systems.

Binary systems provide a unique opportunity to determine the masses of both components. In most HMXBs, the compact object is an X-ray pulsar; pulse-timing analysis is an accurate method to derive the mass-function of the system. Given the radial-velocity curve of the supergiant and an estimate of the system's inclination, the masses of both components can be determined. One should keep in mind, however, that the binary system underwent phases of mass transfer. The situation may occur that the derived mass of the supergiant does not correspond to that of a supergiant with similar spectral type and luminosity that evolved in e.g., a wide binary without mass transfer. For example, in the O7f plus $\mathrm{O} 6 \mathrm{f}$ eclipsing binary $\mathrm{BD}+42^{\circ} 4220$ (V729 Cyg) the stars have almost identical luminosity, but a mass ratio of 3.3 (Massey \& Conti 1977). Probably, the less massive component is a WR star that looks like an $\mathrm{O}$-type star due to accretion of hydrogen from the $\mathrm{O}$ star companion (Vreux 1985). In Table 1 we collected from literature some important parameters of a number of HMXBs with OB supergiant companion, in our galaxy and in the Magellanic Clouds. For most systems the X-ray luminosity is pulsed, identifying the X-ray source as a neutron star; both Cyg X-1 and LMC X-1 are black-hole candidates. The short-period X-ray pulsars are found in systems that contain an accretion disk. In these binaries the supergiant has begun to overflow its Roche lobe. The other supergiants 


\section{TABLE I}

High-Mass X-ray Binaries with supergiant companion in the Galaxy and in the Magellanic Clouds, ordered by the spectral type of the supergiant. The X-ray luminosity is given in units of $10^{36} \mathrm{erg} / \mathrm{s}$. The mass determinations are obtained from: (a) Heap \& Corcoran 1992; (b) Nagase 1989; (c) Gies \& Bolton 1986; (d) Sato et al. 1985. The spectral type of Wray 977 is proposed by Kaper et al. $1994 \mathrm{~b}$.

\begin{tabular}{|c|c|c|c|c|c|c|}
\hline \multicolumn{7}{|c|}{ Galaxy } \\
\hline Name (X-ray ID) & $\begin{array}{l}\text { Sp. } \\
\text { Type }\end{array}$ & $\begin{array}{c}P_{\text {orb }} \\
\text { (days) }\end{array}$ & $\begin{array}{c}P_{\text {pulse }} \\
(\mathrm{sec})\end{array}$ & $\begin{array}{c}L_{X}^{36} \\
(\mathrm{erg} / \mathrm{s})\end{array}$ & $\begin{array}{l}M_{\text {opt }} \\
\left(M_{\odot}\right)\end{array}$ & $\begin{array}{c}M_{X} \\
\left(\mathrm{M}_{\odot}\right)\end{array}$ \\
\hline HD $153919^{a}(1700-37)$ & O6.5 Iaft & 3.41 & & 1.8 & $\sim 52$ & $1.8 \pm 0.4$ \\
\hline V779 Cen $^{b}($ Cen X-3) & O6.5 II-III & 2.089 & 4.84 & 50.6 & $19.8 \pm 4$ & $1.06 \pm 0.5$ \\
\hline V725 Tau $(0535+26)$ & O9.7 IIe & 111 & 104 & 57.1 & & \\
\hline HDE $226868^{c}($ Cyg X-1) & O9.7 Iab & 5.6 & & 21.5 & $>20$ & $>7$ \\
\hline QV Nor ${ }^{b}(1538-52)$ & B0 Iab & 3.73 & 529 & 4.9 & $16.9 \pm 5$ & $1.79 \pm 0.9$ \\
\hline HD $77581^{b}$ (Vela X-1) & B0.5 Ib & 8.96 & 283 & 8.3 & $23.0 \pm 1$ & $1.77 \pm 0.2$ \\
\hline Wray $977^{d}(\mathrm{GX} 301-2)$ & $\mathrm{B} 1.5 \mathrm{Ia}+$ & 41.5 & 696 & 70.3 & $\gtrsim 35$ & \\
\hline$(1145-61)$ & B2 Iae & $10.76 ?$ & 298 & 13.8 & & \\
\hline \multicolumn{7}{|c|}{ Magellanic Clouds } \\
\hline $\mathrm{Sk}^{-\mathrm{Ph}^{b}}(\mathrm{LMC} \mathrm{X}-4)$ & O7 III-V & 1.40 & 13.5 & 410.5 & $14.7 \pm 4$ & $1.38 \pm 0.5$ \\
\hline \#32 (LMC X-1) & O7-9 III & & & 171.1 & & \\
\hline Sk $160^{b}(\mathrm{SMC} X-1)$ & B0 Ib & 3.89 & 0.71 & 514.1 & $16.8 \pm 4$ & $1.06 \pm 0.3$ \\
\hline
\end{tabular}

are close to filling their Roche lobe, which should explain the observed ellipsoidal variations (van Paradijs 1991). The MC sources stand out regarding their high X-ray luminosity. According to radiation-driven wind theory, low metallicity winds have lower outflow velocities due to to absence of driving metal lines, which thus results in a relatively high X-ray luminosity (Eq. 1).

\section{Interaction of X-ray source and stellar wind}

As said, the X-ray luminosity is a measure of the density and velocity of the accreting wind plasma. When a lot of angular momentum is associated with the accreting material, an accretion disk might develop. Especially in systems where the supergiant overflows its Roche-lobe (e.g., Cen X-3), the matter streams to the compact star via a disk. In the other systems (e.g., Vela $\mathrm{X}-1$ ) there is no observational indication for the presence of an accretion disk surrounding the X-ray source. In the latter systems we might therefore expect that the X-ray luminosity directly relates to the local stellar-wind properties. In Fig. 1 we show the X-ray luminosity as a function of time for the system HD 153919 (1700-37). The X-ray luminosity is strongly variable: the flaring occurs on a timescale of hours to days and can reach an amplitude 


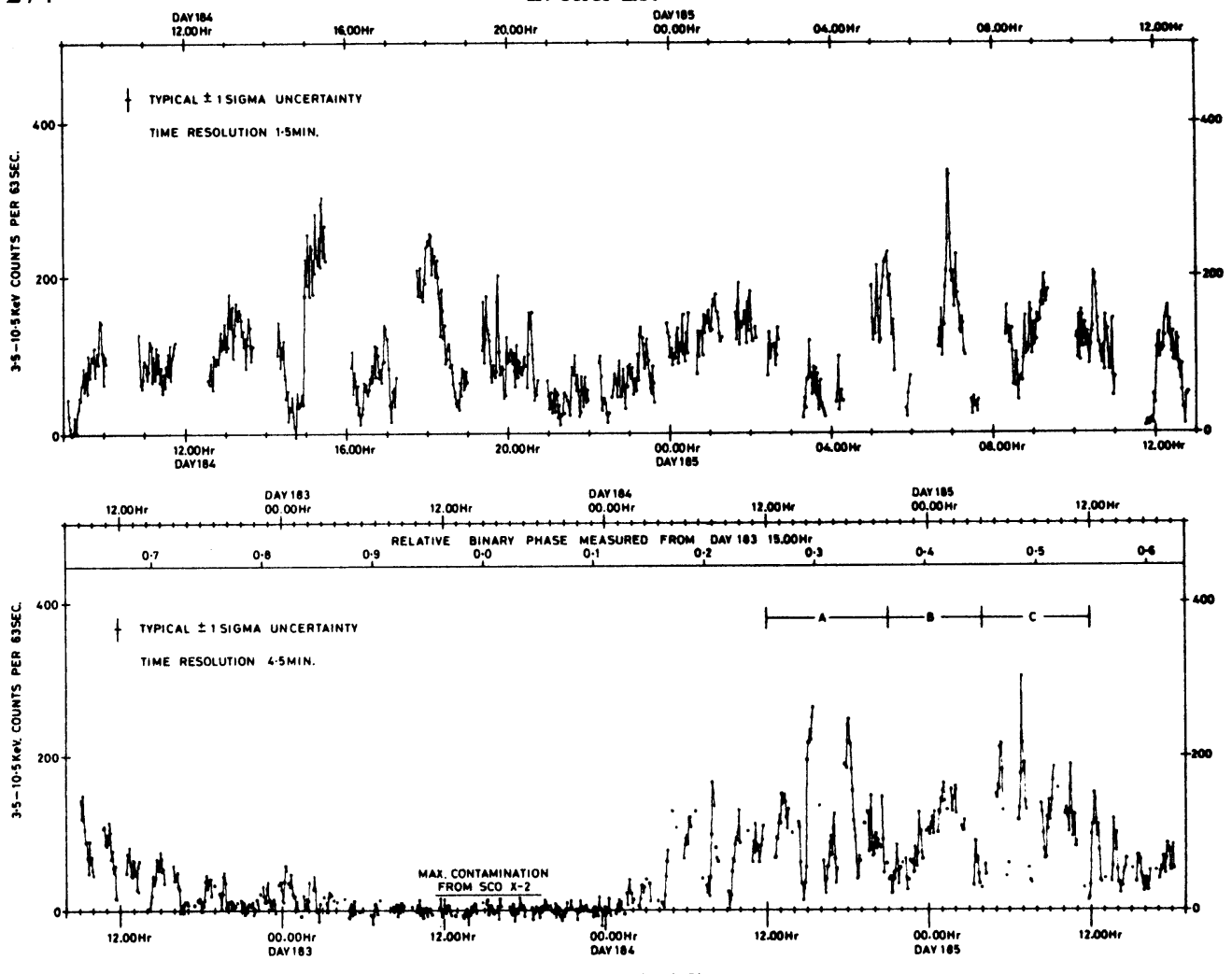

1974 DAY NUMBER (UT.)

Fig. 1. The flaring X-ray luminosity of the HMXB HD 153919 (1700-37) observed in the 3.5-10.5 keV band of the Copernicus satellite (from Mason et al. 1976).

of about a factor of 100 in X-ray intensity. This behaviour is typical for wind-fed HMXBs. Although the accretion mechanism might be intrinsically unstable (e.g., Matsuda et al. 1991), the flaring X-ray luminosity suggests that the accreting medium (i.e., the stellar wind) is inhomogeneous.

\subsection{HatchetT-MCCray efFect}

The X-ray luminosity further ionizes the surrounding medium. A Strömgren zone of higher ionization travels with the $\mathrm{X}$-ray source that orbits the supergiant. Strong UV resonance lines of Si IV, C IV, and N V that are formed in the stellar wind have P-Cygni-type profiles and show large modulations with the orbital period. When the X-ray source is in front of the supergiant, the ions in the line of sight that normally give rise to the blue-shifted absorption part of the profile are ionized away, which results in a strongly diminished P-Cygni absorption. This so-called Hatchett-McCray effect (1977) is observed for Vela X-1, Cyg X-1, SMC X-1, and LMC X-4 in ultraviolet spectra (Hammerschlag-Hensberge 1982). Although Hatchett \& McCray originally made the prediction that this effect should be present in spectra of 

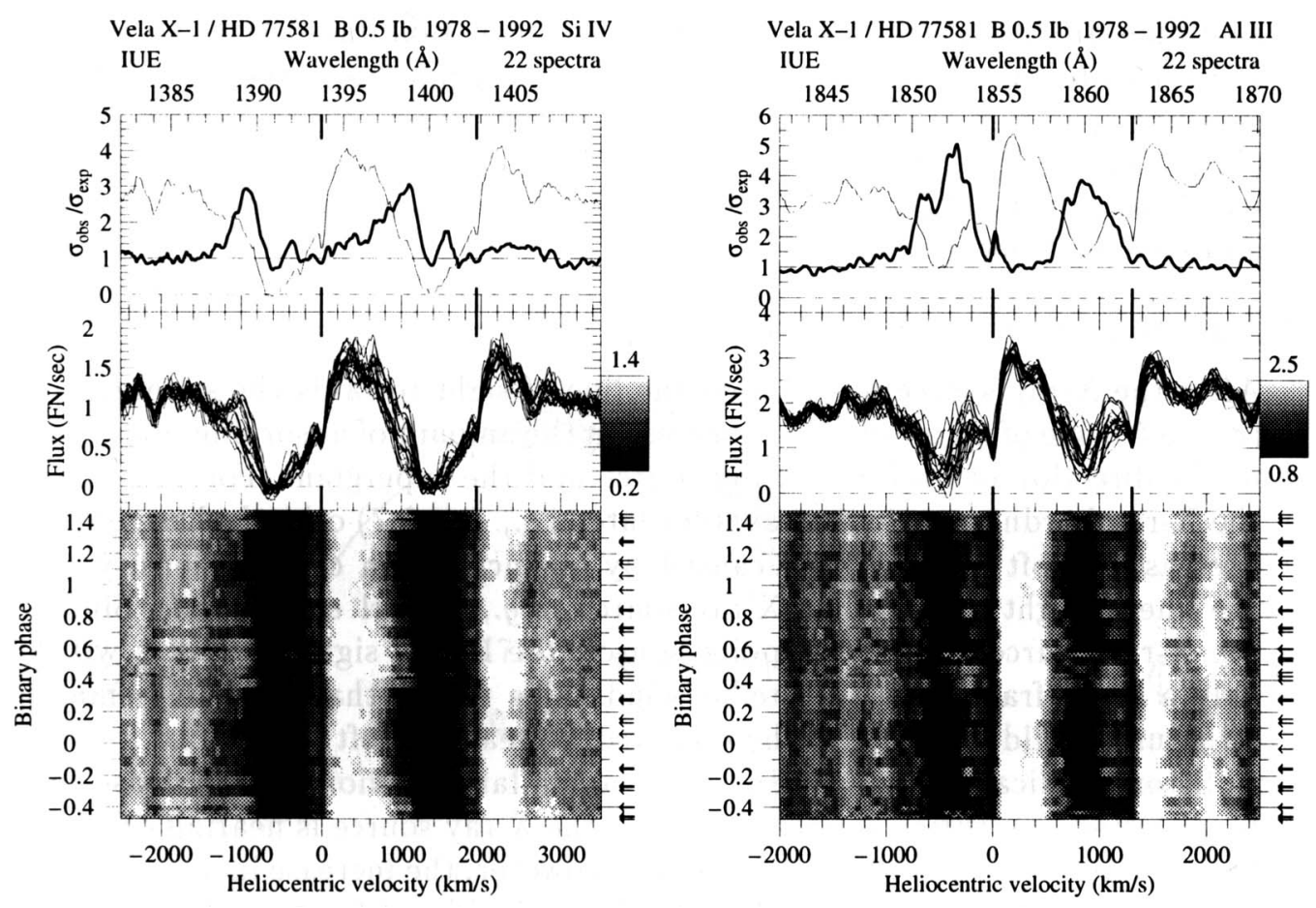

Fig. 2. We present two grey-scale figures showing the HM-effect in the UV resonance lines of Si IV (left) and Al III (right). In the lower panels, the spectra (epoch of observation indicated by an arrow) are stacked on top of each other, orbital phase running upwards. At $\phi=0.5$ the X-ray source is in front of the supergiant which results in a diminished $\mathrm{P}$-Cygni absorption. The thick line in the upper panels is a measure of the significance of variability. Note the occurrence of the HM-effect also at low velocities.

HD 153919, for this system it was not found. A recent study of the HM-effect in high-resolution IUE spectra of HD 77581 (Vela X-1) has shown that the HM-effect sets in already at low $(300-400 \mathrm{~km} / \mathrm{s})$ velocities (Kaper et al. 1993, see Fig. 2), is absent at intermediate velocities in saturated lines, and reaches maximum amplitude at the highest velocities up to $v_{\infty}$. That the UV resonance lines remain saturated at intermediate velocities and not at lower velocities is difficult to explain with a usually adopted monotonically increasing velocity of the stellar wind.

Though the saturation of UV P-Cygni profiles by itself points to a nonmonotonic velocity law (Lucy 1982), the above observations of HD 77581 and the absence of the HM-effect in UV P-Cygni lines of HD 153919 suggest that the stellar winds in these systems are structured (Kaper et al. 1993). An interesting aspect of HD 153919 (1700-37) is the occurence of numerous broad emission lines in the high-resolution ultraviolet spectra that vary in strength and position as a function of orbital phase. These broad emis- 
sion lines are interpreted as Raman scattered emission lines (with the He II $1640 \AA$ transition as the scattering line) that originate from the EUV emission line spectrum (close to the He II $256 \AA$ transition) of the X-ray source (Kaper et al. 1990). Raman scattering has been observed in symbiotic stars which are, like HMXBs, binaries that contain a hot star and a cool star with an extended atmosphere.

\subsection{LATE-PHASE ABSORPTION}

After the X-ray source has crossed the line of sight towards the supergiant $(\phi=0.5)$, one often observes an increase in the amount of absorbing material in the direction of both the X-ray source and the supergiant. For instance, the $\mathrm{X}$-ray hardness ratio increases for late (i.e., $\phi>0.7)$ orbital phases; the decrease in soft X-ray flux is caused by an increase in column density in the line of sight towards the X-ray source (e.g., Haberl et al. 1989). Since the X-ray source is almost a point source, the line of sight intersects with only a small fraction of the stellar wind. This means that relatively small structures could account for the observed decrease in soft X-ray flux.

Strong optical lines (like $\mathrm{H} \alpha$ ) show a similar behaviour: an increase in blue-shifted absorption is observed when the X-ray source is nearing eclipse (e.g., Conti \& Cowley 1975). In this case, however, the increase in absorption must be caused by material that is covering a significant fraction of the much larger supergiant.

Two-dimensional hydrodynamical calculations of Blondin et al. (1990, 1991) in which many effects influencing the wind dynamics around the compact object (gravitation, radiative acceleration, photo-ionization, X-ray heating/cooling, etc.) are included, predict the formation of an accretion wake consisting of dense filaments that trails the X-ray source in its orbit. Depending on the binary separation, a tidal stream may also be present in the system. Blondin et al. (1990) showed that the accretion wake can account for the observed increase in X-ray hardness ratio at late orbital phases. Carlberg (1978), however, argued that an accretion wake around the X-ray source would not be of a sufficient extent to explain the observed increase in absorption in strong optical lines. MacGregor \& Vitello (1982) showed that the acceleration mechanism of the wind is much less efficient if the ionization state of the plasma is so high that the ions which normally provide most of the driving lines are ionized away. As a consequence, in HMXBs the stellar wind inside the ionization zone around the X-ray source is not accelerated; this results in the formation of a strong "photo-ionization" wake at the border of the Strömgren zone (see Fig. 3). This wake is also predicted by the hydrodynamical models of Blondin et al. (1990) for relatively high X-ray luminosities. Recent optical spectroscopic observations of HD 77581 and HD 153919 clearly show the evolution of a blue-shifted absorption component that shifts towards higher velocity with increasing orbital phase (Kaper et al. 


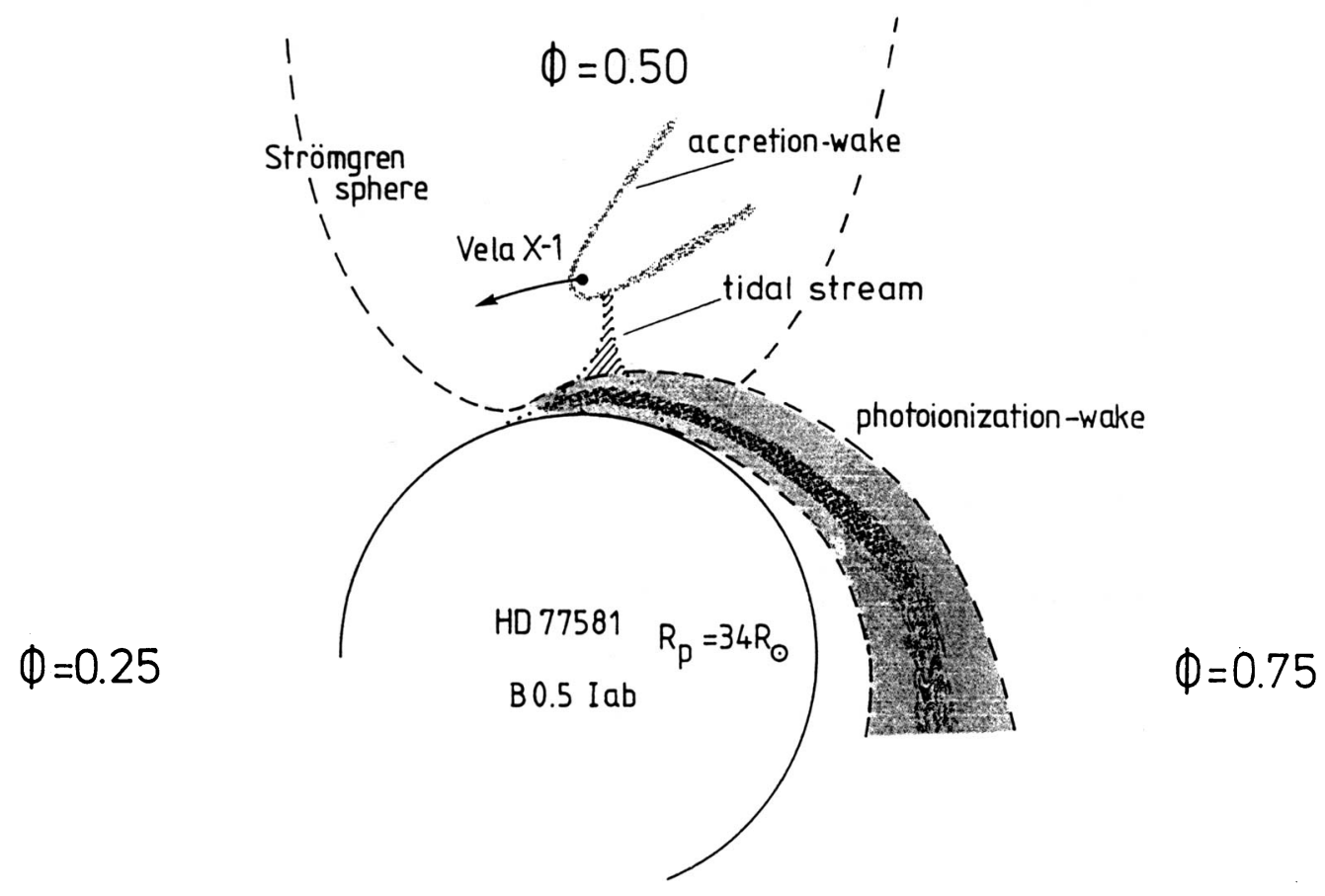

Fig. 3. A sketch of the different structures in the stellar wind of HD 77581 based on observations at different orbital phases of the system. The position of the observer is shown for three orbital phases. X-ray observations indicate the presence of an accretion wake surrounding the compact object. A possible tidal stream is also illustrated in the figure. The position of the photo-ionization wake is based on the observed evolution with orbital phase of the central velocity of the blue-shifted absorption component in the $\mathrm{H} \beta$ line of HD 77581 (from Kaper et al. 1994a).

1994a). These observations are consistent with the presence of an extended photo-ionization wake at the trailing border of the Strömgren zone.

\section{Conclusions}

¿From the above we can conclude that observations of HMXBs reveal important information on stellar-wind structure. Hydrodynamical calculations (e.g., Owocki et al. 1988) have shown that the unstable nature of radiationdriven winds might lead to the development of strong shocks, i.e., a clumpy wind structure. Accretion of this inhomogeneous wind should naturally result into a strongly variable $\mathrm{X}$-ray luminosity. The $\mathrm{X}$-rays further ionize the surrounding medium; this Strömgren zone is observed through the orbital modulation of UV resonance lines. Detailed inspection of the HM-effect leads 
to the conclusion that the velocity structure of these stellar winds is nonmonotonic (i.e., shocked). Inside the Strömgren zone radiative acceleration is quenched and the stagnant flow inside this zone collides with the rapidly accelerating wind at the trailing border. The "colliding winds" form a strong extended shock that can account for the observed late-phase absorption in optical spectra. Apart from the photo-ionization wake, an accretion wake (causing the observed change in $\mathrm{X}$-ray hardness ratio) and/or a tidal stream may be present in the system.

The stellar-wind structure, the radiative acceleration, and the accretioninduced X-ray luminosity are all interrelated and variable in time. The complexity of the different interactions can only be solved by simultaneous observation of the relevant parameters and detailed modelling using sophisticated time-dependent hydrodynamical calculations. By doing this, important progress will be made in the understanding of this challenging aspect of radiation hydrodynamics.

\section{Acknowledgements}

The author acknowledges financial support from the Local Organizing Committee and the Leids Kerkhoven-Bosscha Fonds.

\section{References}

Blaauw, A. 1961, BAN 15, 265

Blondin, J.M., Kallman, T:R., Fryxell, B.A., Taam, R.E. 1990, ApJ 356, 591

Blondin, J.M., Stevens, I.R., Kallman, T.R. 1991, ApJ 371, 684

Bondi, H., Hoyle, F. 1944, MNRAS 104, 273

Carlberg, R.G. 1978, PhD thesis, Univ. of British Columbia

Castor. J.I., Abbott, D.C., Klein, R.K. 1975, ApJ 195, 157

Conti, P.S., Cowley, A.P. 1975, ApJ 200, 133

Gies D.R., Bolton C.T. 1986, ApJ 304, 371

Haberl F., White N.E., Kallman T.R. 1989, ApJ 343, 409

Hammerschlag-Hensberge, G. 1980, in: Proc. $2^{\text {nd }}$ European IUE Conf., ESA SP-157, p. lix-lxv

Hatchett S., McCray R. 1977, $A p J$ 211, 552

Heap S.R., Corcoran M.F. 1992, ApJ 387, 340

Hellings 1983, ApJ Suppl. 96, 37

Kaper, L., Hammerschlag-Hensberge, G., Takens, R.J. 1990, Nature 347, 652

Kaper, L., Hammerschlag-Hensberge, G., van Loon, J.Th. 1993, $A \& A$ 279, 485

Kaper, L., Hammerschlag-Hensberge, G., Zuiderwijk, E.J. 1994a, $A \mathscr{E} A$ in press.

Kaper, L. et al. 1994b, A\&A (Letters) submitted

Kudritzki, R.P., Hummer, D.C. 1990, Ann. Rev. A\& A 28, 303

Lucy, L.B. 1982, ApJ 255, 278

Lucy, L.B., Solomon, P.M. 1970, $A p J$ 159, 879

MacGregor K.B., Vitello P.A.J. 1982, $A p J$ 259, 267

Mason, K.O., Branduardi, G., Sanford, P.W. 1976, ApJ (Letters) 203, L29

Massey, P., Conti, P.S. 1977, ApJ 218, 431

Matsuda, T., Sekino, N., Sawada, K., Shima, E., Livio, M., Anzer, U., Börner, G. 1991, $A \mathscr{G} A$ 248, 301 
Nagase, F. 1989, PASJ 41, 1

Owocki, S.P., Castor, J.I., Rybicki, G.B. 1988, ApJ 335, 914

Pols, O.R. 1993, PhD thesis, University of Amsterdam

Sato N., Nagase, F., Kawai, N., Kelley, R.L., Rappaport, S., White, N.E. 1985, ApJ 304, 241

van den Heuvel, E.P.J. 1994, in: Interacting Binaries, Saas-Fee Advanced Course 22 (Berlin: Springer), p. 263

van den Heuvel, E.P.J., Rappaport, S.A. 1987, in: Slettebak \& Snow (eds.), Physics of Be Stars, p. 291

van Paradijs, J.A. 1991, in: J. Ventura \& D. Pines (eds.), Neutron Stars: Theory and Observations, NATA ASI-Series C 344 (Dordrecht: Kluwer), p. 245

Vreux, J.M. 1985, $A \& A$ 143, 209

\section{DISCUSSION:}

Stevens: The hydrodynamic models of Blondin et al. (1990) have an intrinsic method of producing flaring behaviour, namely that an instability associated with the accretion naturally causes X-ray flares. Non-monotonic winds may also play a role in flaring, but is probably not the only effect.

Conti: Can you say anything quantitative about the relationship between X-ray flaring in highmass X-ray binaries and "blob" behaviour?

Kaper: The X-ray luminosity is proportional to $\rho / \mathrm{v}^{3}$; time-dependent hydrodynamical models of radiation-driven winds by Owocki and collaborators predict the development of shocks in the wind. The resulting wind structure is that of alternating high-density, low-velocity regions and low-density, high-velocity regions. These properties of a clumpy wind naturally cause a strongly variable X-ray luminosity, and easily account for the observed amplitude in X-ray flux.

Koenigsberger: You presented a figure where the Stromgren zone is much larger than the dimensions of the (accretion) wake. To what ionization stage does this Strömgren zone correspond?

Kaper: First of all, the figure I showed was just a sketch, intended to show the different areas in the wind surrounding the X-ray source. The sketched size of the Stromgren zone should correspond to the zone where the radiative acceleration of the wind has quenched due to the higher ionization state of the plasma. At the border of this zone the fast wind collides with the stagnant wind inside the Strömgren zone and forms the photo-ionization wake.

Niemela: Is any of these HMXBs related with a supernova ramnant?

Polcaro: The HMXB system HDE $245770(\mathrm{~A} 0535+26)$ is associated with an old supernova remnant; the age of the SNR is compatible with the age of the neutron star and the radio distance of the SNR is similar to that of HDE 245770 (a 09.5 Ve star), as obtained from the UV extinction (by zeroing the $2200 \AA$ absorption). There are a few other cases, but less convincing. Cherepashchuk: Did you try to investigate rapid X-ray variability of the Cyg X-3 binary system to check the clumping structure of the wind of the WR companion?

Kaper: No, I did not; the X-ray data, however, should be available to make such a check, although the length of the dataset in time should be sufficiently long (a flow timescale in the wind is on the order of hours to a day) to draw any conclusions. 